Research Article

\title{
Development and Validation of Rapid 3D Radiation Field Evaluation Technique for Nuclear Power Plants
}

\author{
Weifeng Lyu $\mathbb{D}^{\mathrm{D}},{ }^{1}$ Wenwang Ran $\mathbb{D}^{1},{ }^{1}$ Jie Liu, ${ }^{1}$ Jingyu Zhang, ${ }^{2}$ Shaohua Tang, ${ }^{1}$ and Jun Xiong ${ }^{1}$ \\ ${ }^{1}$ State Key Laboratory of Nuclear Power Safety Monitoring Technology and Equipment, Shenzhen 518172, China \\ ${ }^{2}$ School of Nuclear Science and Engineering, North China Electric Power University, Beijing 102206, China \\ Correspondence should be addressed to Weifeng Lyu; liweifeng1000@163.com
}

Received 8 March 2020; Accepted 15 May 2020; Published 3 June 2020

Academic Editor: Rafa Miró; rmiro@upv.es

Copyright (c) 2020 Weifeng Lyu et al. This is an open access article distributed under the Creative Commons Attribution License, which permits unrestricted use, distribution, and reproduction in any medium, provided the original work is properly cited.

Rapid 3D radiation field evaluation is the key point of occupational dose optimization for design and operation of nuclear power plant. Based on the requirement analysis from designers and operators of nuclear power plant, three key technical issues are identified and solved through the development of the RPOS system, which are rapid calculation of 3D radiation field, reconstruction of the calculated 3D radiation field based on measured data, and occupational dose optimization based on 3D radiation field. Operational measurements of dose rate from in-service nuclear power plants are used to test the RPOS system, which shows that accurate 3D radiation field can be rapidly generated by the RPOS system and effectively used on the occupational dose optimization for on-site workers. The applications of the established rapid 3D radiation field evaluation technique on HPR1000 unit design provide evidence on its feasibility in a large scale, the improvement of radiation protection design efficiency and the enhancement of ALARA assessment and justification for nuclear power plants.

\section{Introduction}

During the present radiological protection design and operation of nuclear power plant, the dose rates of several characteristic points are usually referenced as the design basis. Hence, the occupational dose optimization and ALARA improvement are limited due to the lack of fine and accurate dose rate radiation field. The existing codes for the generation of dose rate radiation field in specific cases are not sufficient to apply in overall nuclear power plant design due to their efficiency and accuracy.

Many research institutions have carried out various studies on the calculation of 3D radiation field and its applications. The fusion digital simulation (FDS) team of the Chinese Academy of Sciences has calculated the 3D dose rate of several research nuclear devices based on the Monte Carlo method and achieved the dose assessment based on radiation field and its visualization $[1,2]$. The SCK.CEN has developed the VISIPLAN 3D ALARA planning tool based on the point kernel integration method to evaluate the dose due to external gamma exposure, including simulations of work scenarios, worker positions, and source distribution in a 3D environment [3]. Some research institutions have introduced new technologies, such as virtual reality (VR), to support 3D radiation field display. Embedded with VR, tasks based on 3D radiation field, such as maintenance scheme optimization, cost savings, and virtual training, can be achieved [4-8]. Radiation field technique is also applied worldwide in decommissioning scenarios of research nuclear facilities [9-13], and several reconstruction methods of radiation field are also proposed [14-16]. Although 3D radiation field calculation has been a common subject of research worldwide, practicable rapid 3D radiation field evaluation technique still needs to be developed and applied on large-scale radiological protection design and operation of nuclear power plant. Moreover, the development of accurate and applicable system is important for occupational dose optimization and ALARA improvement to promote radiological safety for workers.

\section{Development of Rapid 3D Radiation Field Evaluation Technique}

From the viewpoint of operators of in-service nuclear power plants, a technique which allows the visualization of pipes, 
equipment, and walls combined with the dose rate radiation field in any area of the plants is required to evaluate the exposure and familiarize the workers with the radiation distribution and layout of the workplace before the actual operations, such as equipment maintenance and equipment replacement. In addition, the relative error between the displayed $3 \mathrm{D}$ radiation field and the actual value is required to be within an acceptable range. The collective dose of the nuclear power plant can be effectively reduced by applying this technique and further reduced if an automatic dose optimization technique is available.

From the viewpoint of designers of nuclear power plant, the key requirement for this technique is the rapid generation of $3 \mathrm{D}$ dose rate radiation field of the plants, which allows radiological protection engineers to check the shielding design and find out the shielding weakness in an efficient manner. A supporting tool of the 3D radiation field visualization also should be considered to meet this requirement.

Based on the above requirement of designers and operators of nuclear power plant, the following key functions should be implemented in the rapid $3 \mathrm{D}$ radiation field evaluation technique: (a) rapid and accurate generation of $3 \mathrm{D}$ radiation field; (b) reconstruction of $3 \mathrm{D}$ radiation field based on dose rate measurements; (c) application of dose optimization, such as route and operation optimization.

Apart from the above three functions, the technique of visualization of geometric model combined with $3 \mathrm{D}$ radiation field is required to facilitate the application of $3 \mathrm{D}$ radiation field evaluation technique.

\subsection{Rapid and Accurate Generation of 3D Radiation Field.} The accuracy of the 3D radiation field is highly dependent on the source term and the dose rate calculation method used. Hence, selection of a suitable dose rate calculation algorithm or commercial code is one of the key points to the rapid and accurate generation of $3 \mathrm{D}$ radiation field.

The algorithm or code selected should meet the following requirements: (a) capable to deal with the calculation of complex geometries as it is generally the case in nuclear power plant; (b) capable to deal with the calculation of multiple source terms; (c) capable to deal with the calculation of neutron and photon transport as neutron shielding is required in nuclear power plant design.

SuperMC code $[17,18]$ is selected as the calculation code of $3 \mathrm{D}$ radiation field based on the above requirements. SuperMC is a Monte Carlo code developed by the FDS team of the Chinese Academy of Sciences and capable of carrying out neutron and photon transport calculation in complex geometries.

The input parameters of $3 \mathrm{D}$ radiation field calculation are geometry, material, and source term. Therefore, the following functions are necessary in order to achieve the rapid generation of the $3 \mathrm{D}$ radiation field: (a) a rapid geometry and material input interface; (b) a rapid source term input interface. The solutions for each function are detailed below.

Manual import of geometry is inefficient and cumbersome due to the significant amount of equipment and pipes in nuclear power plant and the complexity of layout. An interface module to import and automatically convert the geometry and material information into SuperMC calculation input file is therefore implemented for $3 \mathrm{D}$ radiation field calculation. The module is a secondary development of MCAM code which is the input module of SuperMC code by integration of commercial codes Spaceclaim [19], which supports the common 3D model format widely used in the nuclear industry, such as PDMS, CATIA, and SolidWorks. The typical geometry importation process is as follows: (a) for the first, ".stp" format file is exported from PDMS code by MEI-O interface module; (b) then SpaceClaim code is used to treat the ".stp" format file, and ".sat" format file is exported from SpaceClaim; (c) finally ".sat" format file is imported to SuperMC code by MCAM code.

As another crucial input for $3 \mathrm{D}$ radiation field calculation, the radioactive source terms presented in the controlled area of nuclear power plant are mainly the radioactive fluids and corrosion products in the equipment and pipes. For nuclear power plants designed by China General Nuclear Power Corporation (CGN), the source terms of equipment and pipes are calculated by in-house developed code CPDS2.0. An interface module which can convert the source term results of CPDS2.0 code into SuperMC calculation input file is implemented. In addition, the interface module which allows using measurement data of source term as an input is also considered for general application of $3 \mathrm{D}$ radiation field calculation.

2.2. 3D Radiation Field Reconstruction. Inevitable measurement errors of input data and errors of dose rate calculation will cause the generated $3 \mathrm{D}$ radiation field to deviate from the actual radiation field. Reconstruction of dose rate calculation results based on the actual measured data of several characteristic points in the field can significantly reduce the deviation.

According to the radiation field calculation, radioactive source and geometric model are the main factors to the results obtained. As the geometric model is relatively accurate, the uncertainty introduced by geometric model is negligible. Therefore, $3 \mathrm{D}$ radiation field reconstruction carried out with respect to the radioactive source will improve the results.

When multiple radioactive sources are present, the dose rate at a certain point in the area is the sum of the dose rate caused by each radioactive source individually. Assuming there are $p$ sources in the area, the actual measured data for a specified measurement point $\mathrm{A}$ in the area is in linear relationship with calculation results of dose rate of each source, as shown:

$$
Y_{\mathrm{A}}=\beta_{1} \cdot X_{\mathrm{A} 1}+\beta_{2} \cdot X_{\mathrm{A} 2}+\cdots+\beta_{p} \cdot X_{\mathrm{AP}},
$$

where $Y_{\mathrm{A}}$ is the actual measured data of dose rate at point $\mathrm{A}$, $\mathrm{mSv} / \mathrm{h} ; \beta_{j}$ is the correlation factor for the $j$-th source, $j=1, \ldots, p$; and $X_{\mathrm{A} j}$ is the theoretical calculation value of dose rate at point $A$ caused by the $j$-th source, which is calculated by SuperMC code based on assumed theoretical source term and real geometry model, $\mathrm{mSv} / \mathrm{h}$. The theoretical source term used is derived based on conservative assumptions during the design stage of the nuclear power plant. 
Furthermore, assuming there are $n$ measurement points in the compartment, equation (1) can be rewritten in a matrix form as follows:

$$
Y=X \cdot B
$$

where

$$
\begin{aligned}
Y & =\left[\begin{array}{lllll}
y_{1} & y_{2} & y_{3} & \cdots & y_{n}
\end{array}\right]^{\mathrm{T}}, \\
X & =\left[\begin{array}{ccccc}
x_{11} & x_{12} & x_{13} & \cdots & x_{1 p} \\
x_{21} & x_{22} & x_{23} & \cdots & x_{2 p} \\
\cdots & \cdots & \cdots & \cdots & \cdots \\
x_{n 1} & x_{n 2} & x_{n 3} & \cdots & x_{n p}
\end{array}\right], \\
B & =\left[\begin{array}{lllll}
\beta_{1} & \beta_{2} & \beta_{3} & \cdots & \beta_{p}
\end{array}\right]^{\mathrm{T}} .
\end{aligned}
$$

If theoretical calculation values of dose rate at each point are known for every radioactive source in the area, the problem of the $3 \mathrm{D}$ radiation field reconstruction is converted into solving vector $B$, which equals to minimize the following equation:

$$
S(B)=\sum_{i=1}^{n}\left(\frac{y_{i}-\beta_{1} \cdot x_{i 1}-\cdots-\beta_{p} \cdot x_{i p}}{y_{i}}\right)^{2},
$$

where $S(B)$ is the sum of the relative variance of the measured dose rate and the reconstructed dose rate for all measurement points;

By deriving $S(B)$ with respect to $\beta_{j}$ and making it equal to zero, $p$ linear equations can be derived:

$$
\begin{aligned}
& \beta_{1} \cdot \sum_{i=1}^{n} x_{i j}^{\prime}+\cdots+\beta_{p} \cdot \sum_{i=1}^{n} x_{i p}^{\prime}=\sum_{i=1}^{n} y_{i}^{\prime}, \\
& \beta_{1} \cdot \sum_{i=1}^{n} x_{i 1}^{\prime} \cdot x_{i j}^{\prime}+\cdots+\beta_{p} \cdot \sum_{i=1}^{n} x_{i j}^{\prime} \cdot x_{i p}^{\prime}=\sum_{i=1}^{n} y_{j}^{\prime} \cdot y_{i}^{\prime}, \\
& j=1, \ldots, p, \\
& x_{i j}^{\prime}=\frac{x_{i j}}{y_{i}}, \\
& y_{i}^{\prime}=\frac{y_{i}}{y_{i}}=1 .
\end{aligned}
$$

The above equations can be expressed in the matrix form as follows:

$$
X^{\prime T} \cdot X^{\prime} \cdot B=X^{\prime T} \cdot Y^{\prime}
$$

then,

$$
B=\left(X^{\prime T} \cdot X^{\prime}\right)^{-1} \cdot X^{\prime T} \cdot Y^{\prime}
$$

As a result, the dose rate of the points without measured data are corrected using equation (1) with the solved $\beta_{j}$ in equation (7), and the reconstructed radiation field can be obtained.

2.3. Application of Dose Optimization Based on 3D Radiation Field. According to operational practice of in-service nuclear power plants in China, a typical case of occupational dose optimization is to optimize the working route in a single radiological compartment with multiple unfixed operating positions.

The process of a typical worker operation in a radioactive compartment can be described in the following three steps: (a) the worker enters the compartment from the start point $\mathrm{A}$ and arrives at the operating point $\mathrm{B}$; (b) the worker performs operations at operating point $\mathrm{B}$; (c) the worker departs from operation point $\mathrm{B}$ and leaves the compartment via the end point $C$.

The total dose received by workers during this process can be calculated by the following equation:

$$
A=\bar{D}_{\mathrm{A}-\mathrm{B}} \cdot t_{\mathrm{A}-\mathrm{B}}+D_{\mathrm{B}} \cdot t_{\mathrm{B}}+\bar{D}_{\mathrm{B}-\mathrm{C}} \cdot t_{\mathrm{B}-\mathrm{C}} \cdot
$$

When using discrete grids to represent the $3 \mathrm{D}$ dose rate field, equation (8) can be rewritten as follows:

$$
A=\sum_{i=\mathrm{A}}^{\mathrm{B}} \frac{D_{i} \cdot l_{i}}{v}+D_{\mathrm{B}} \cdot t_{\mathrm{B}}+\sum_{i=\mathrm{B}}^{\mathrm{C}} \frac{D_{i} \cdot l_{i}}{v},
$$

where $A$ is the total dose received by workers, $m S v ; \bar{D}_{\mathrm{A}-\mathrm{B}}$ is the average effective dose rate from start point $A$ to operating point $\mathrm{B}, \mathrm{mSv} / \mathrm{h} ; t_{\mathrm{A}-\mathrm{B}}$ is the time taken from start point $\mathrm{A}$ to operating point $\mathrm{B}, \mathrm{h} ; D_{\mathrm{B}}$ is the effective dose rate at operating point $\mathrm{B}, \mathrm{mSv} / \mathrm{h} ; t_{\mathrm{B}}$ is the operating time at operating point $\mathrm{B}$, $\mathrm{h} ; \bar{D}_{\mathrm{B}-\mathrm{C}}$ is the average effective dose rate from the operating point $\mathrm{B}$ to the end point $\mathrm{C}, \mathrm{mSv} / \mathrm{h} ; t_{\mathrm{B}-\mathrm{C}}$ is the time from operating point $\mathrm{B}$ to end point $\mathrm{C}, \mathrm{h} ; D_{i}$ is the effective dose rate in grid cell $i, \mathrm{mSv} / \mathrm{h} ; v$ is the workers' moving speed, $\mathrm{m} /$ $\mathrm{h} ; l_{i}$ is the side length of grid cell $i, \mathrm{~m}$.

Since the $3 \mathrm{D}$ radiation field is defined by discrete grids to facilitate the display and data storage, the travel route of workers can be abstracted as a set of connected grids. The purpose of the dose optimization algorithm for single operation in the radioactive compartment is to find out the grid combination with the smallest total dose when the start point, the operating point, and the end point are given. Considering that workers have a certain volume and some area in compartment is not accessible, workers are modeled as cuboid in dose optimization calculation. All realistic routes are determined through free movement and collision check of the worker model in the compartment model, thus completing the division of the discrete grid definition.

After comparison of various algorithms, the genetic algorithm is selected to solve the above optimization problem. The elements of the genetic algorithm include coding rules, initial population generation, fitness functions, various types of operators, and termination conditions. In the algorithm construction, according to the characteristics of the operation dose optimization calculation in the $3 \mathrm{D}$ radiation field, the classic elements of the genetic algorithm are correspondingly chosen or adjusted [20].

\section{Development and Validation of 3D Radiation Field Evaluation System}

3.1. RPOS System Architecture. Based on the research on rapid $3 \mathrm{D}$ radiation field evaluation technique, the $3 \mathrm{D}$ radiation field evaluation system (RPOS) with the following six modules is 


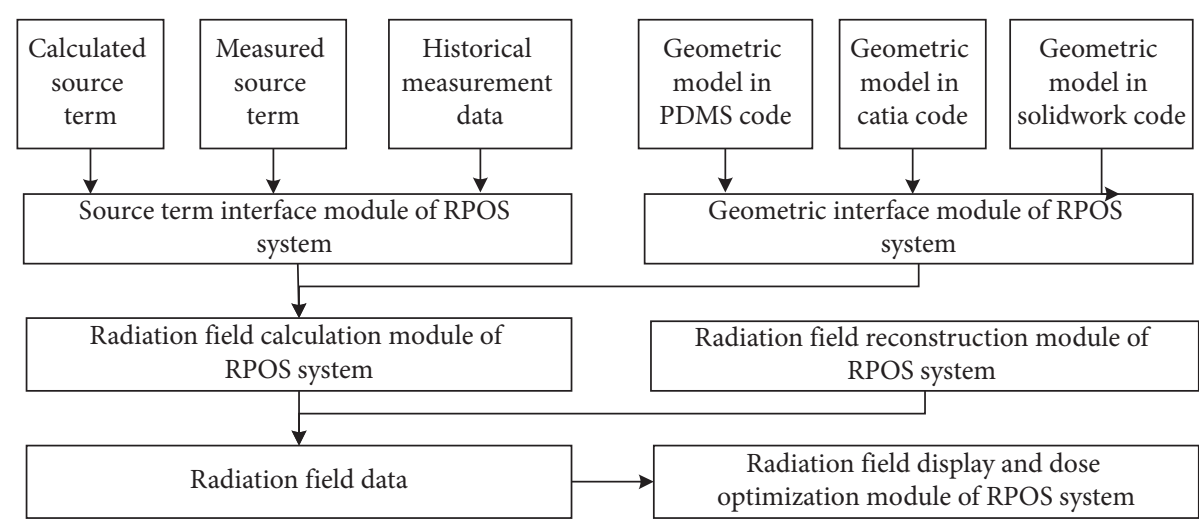

Figure 1: The system architecture of RPOS system.

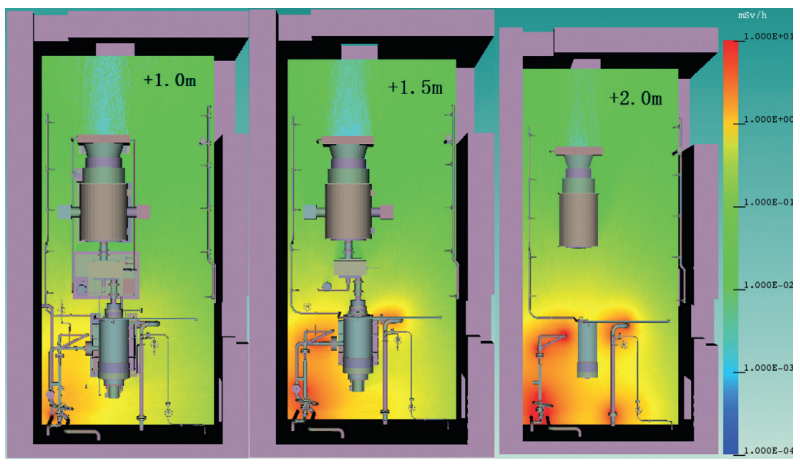

FIgURE 2: Theoretical 3D radiation field of the typical radioactive compartment.

developed: (a) 3D radiation field calculation module; (b) geometric interface module; (c) source term interface module; (d) $3 \mathrm{D}$ radiation field reconstruction module; (e) 3D radiation field display module; (f) 3D radiation field dose optimization module.

The system architecture and relationship between six modules of the RPOS system is shown in Figure 1.

\subsection{Validation of Rapid 3D Radiation Field Calculation and} Display. The 3D radiation field of a typical radiological controlled compartment in a pressurized water reactor plant during power operation is calculated and displayed by RPOS system based on theoretical source term during the plant design stage. The top view of the $3 \mathrm{D}$ dose radiation field at three elevations of $+1.0 \mathrm{~m},+1.5 \mathrm{~m}$, and $+2.0 \mathrm{~m}$ is shown in Figure 2. Main radioactive sources in this compartment are the charging pump and its connected pipes to the chemical and volume control system. According to the benchmark validation of SuperMC code on radiation field calculation $[17,18]$, SuperMC code is applicable for radiation field calculation of the nuclear power plant. The statistical variance of radiation field calculation can be reduced to lower than $1 \%$ by applying sufficient samples and variance reduction techniques.

3.3. Validation of 3D Radiation Field Reconstruction. The 3D radiation field reconstruction module is validated by using the dose rate data measured in the same compartment

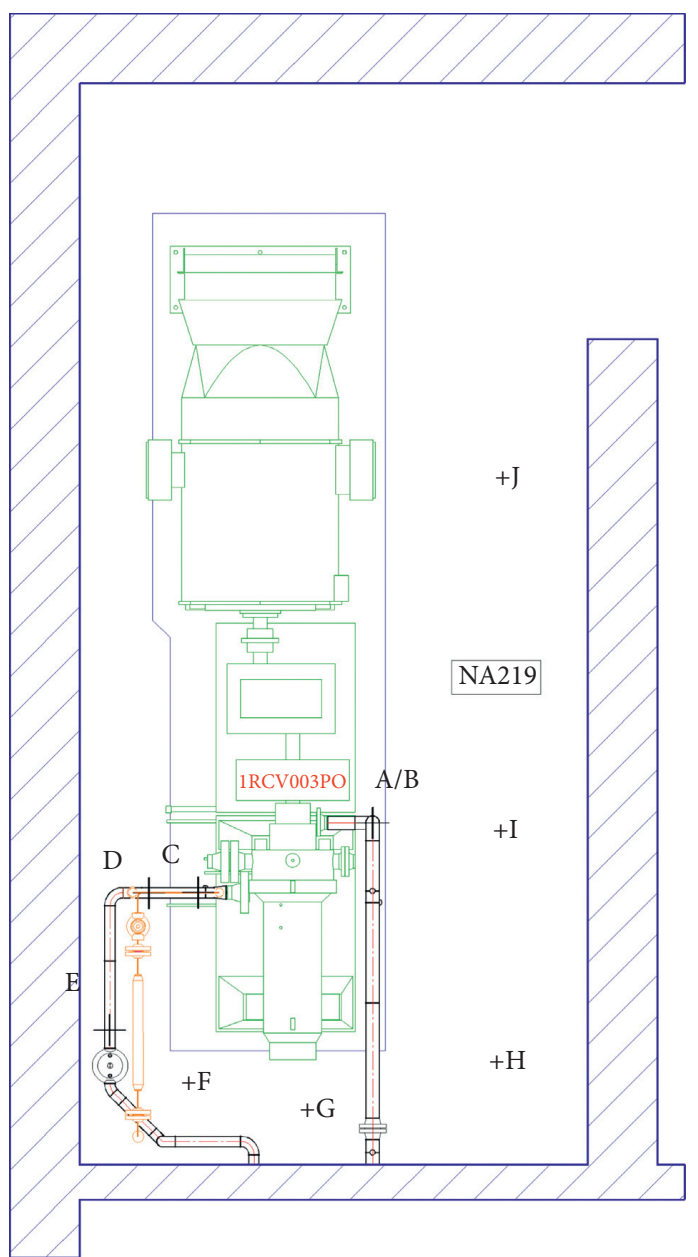

Figure 3: Positions of dose rate measurement points in selected typical radiological compartment.

descripted in Section 3.2. A total number of 10 measurement points $(\mathrm{A} \sim \mathrm{J})$ are set, of which 5 points $(\mathrm{A} \sim \mathrm{E})$ are used for radiation field reconstruction and 5 points $(\mathrm{F} \sim \mathrm{J})$ are used for reconstructed radiation field validation. Locations of the measurement points in the area are shown in Figure 3, and the detailed information is listed in Table 1.

In order to reduce the error introduced by the measurement process, each point is measured for 7 times independently, and 
TABLE 1: Information of dose rate in the selected typical radiological compartment.

\begin{tabular}{|c|c|c|c|c|c|c|c|c|c|}
\hline \multirow[t]{2}{*}{ Measurement point } & \multicolumn{3}{|c|}{$\begin{array}{l}\text { Global coordinates relative } \\
\text { to the center of the core }(\mathrm{cm})\end{array}$} & \multicolumn{6}{|c|}{ Measured dose rate $(\mathrm{mSv} / \mathrm{h})$} \\
\hline & $x$ & $y$ & $z$ & 1 & 2 & 3 & 4 & 5 & Average \\
\hline $\mathrm{A}$ & 2764 & 1342 & 132 & 1.123 & 0.855 & 0.917 & 0.864 & 0.866 & 0.925 \\
\hline B & 2764 & 1342 & 165 & 0.945 & 0.972 & 1.083 & 0.986 & 1.014 & 1.000 \\
\hline $\mathrm{C}$ & 2562 & 1296 & 156 & 1.221 & 1.082 & 1.025 & 1.233 & 1.064 & 1.125 \\
\hline $\mathrm{D}$ & 2587 & 1277 & 128 & 1.425 & 1.259 & 1.315 & 1.225 & 1.276 & 1.300 \\
\hline $\mathrm{E}$ & 2506 & 1220 & 129 & 0.482 & 0.525 & 0.514 & 0.543 & 0.561 & 0.525 \\
\hline F & 2590 & 1150 & 50 & 0.195 & 0.183 & 0.176 & 0.192 & 0.179 & 0.185 \\
\hline $\mathrm{G}$ & 2690 & 1150 & 50 & 0.112 & 0.089 & 0.095 & 0.106 & 0.088 & 0.098 \\
\hline $\mathrm{H}$ & 2860 & 1150 & 50 & 0.052 & 0.046 & 0.058 & 0.051 & 0.048 & 0.051 \\
\hline I & 2860 & 1340 & 50 & 0.033 & 0.038 & 0.041 & 0.031 & 0.032 & 0.035 \\
\hline $\mathrm{J}$ & 2860 & 1730 & 50 & 0.005 & 0.009 & 0.01 & 0.006 & 0.005 & 0.007 \\
\hline
\end{tabular}

the arithmetically average is calculated after eliminating the maximum and minimum measurement. The theoretical 3D radiation field is reconstructed by using the measurement dose rate of point $\mathrm{A} \sim \mathrm{E}$. The reconstructed radiation field of selected typical radioactive compartment is shown in Figure 4.

The reconstructed $3 \mathrm{D}$ radiation field is validated using the measurement data of point $\mathrm{F} \sim \mathrm{J}$. The relative errors of five points are shown in Table 2, and conclusions can be got that the relative error of all points is below or around 30\%, which shows the accuracy fulfills the engineering requirement in most cases.

3.4. Validation of Dose Optimization. The dose optimization function of the RPOS system is validated by theoretical calculation in a typical operation situation in the nuclear power plant. In the validation case, a typical $3 \mathrm{D}$ radiation field with four possible points (B1, B2, B3, and B4) for a fixed operation are defined as well as the start point $A$ and the end point C (Figure 5). Figure 5 shows an example of compartment for the application of dose optimization. Equipment, barrier, and radioactive sources are represented by pink squares.

The input data and total dose under the four operating schemes calculated by genetic algorithm are listed in Tables 3 and 4. According to the results, the most optimized total dose is reached to be $3.40 \mathrm{mSv}$ when selecting the scheme of operating point $\mathrm{B} 1$.

\section{Application of RPOS System on HPR1000 Unit Design}

During the radiation protection design of HPR1000 unit, the RPOS system is widely used to improve the efficiency and the dose optimization demonstration in the following three specific areas: (a) fine 3D radiation field generation and display; (b) shielding evaluation of labyrinth and door; (c) inspection of radiation streaming and shielding weakness.

4.1. Fine 3D Radiation Field Generation and Display. The fine $3 \mathrm{D}$ radiation field of a typical radiological controlled compartment for HPR1000 during power operation is

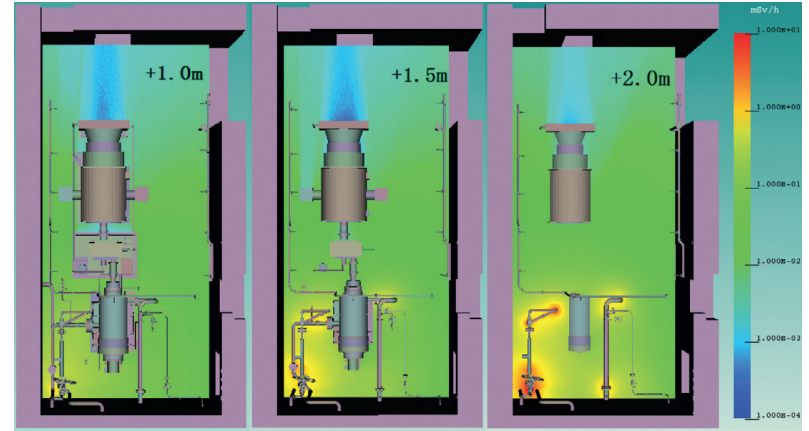

FIGURE 4: Reconstructed 3D radiation field of the selected typical radiological compartment.

TABLE 2: Error analysis of reconstructed 3D radiation field.

\begin{tabular}{lccc}
\hline $\begin{array}{l}\text { Dose } \\
\text { point }\end{array}$ & $\begin{array}{c}\text { Reconstructed dose } \\
\text { rate } X(\mathrm{mSv} / \mathrm{h})\end{array}$ & $\begin{array}{c}\text { Measured dose } \\
\text { rate } Y(\mathrm{mSv} / \mathrm{h})\end{array}$ & $\begin{array}{c}\text { Relative error } \\
((X-Y) / Y)(\%)\end{array}$ \\
\hline $\mathrm{F}$ & 0.224 & 0.185 & 21.15 \\
$\mathrm{G}$ & 0.114 & 0.098 & 16.57 \\
$\mathrm{H}$ & 0.065 & 0.051 & 26.87 \\
$\mathrm{I}$ & 0.046 & 0.035 & 30.52 \\
$\mathrm{~J}$ & 0.009 & 0.007 & 29.30 \\
\hline
\end{tabular}

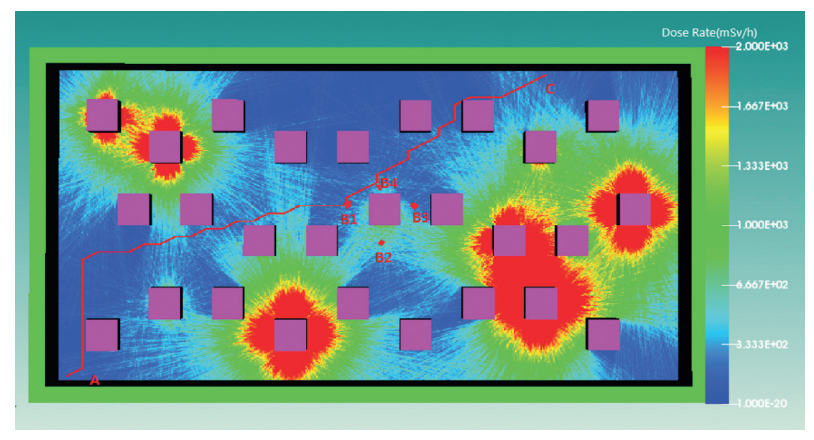

FIgURE 5: Schematic diagram of the optimized operation scheme.

generated and displayed by the RPOS system (Figure 6). The MEI-O interface module of the PDMS code, the MCAM code, and the SpaceClaim code are used subsequently to prepare the geometry and material input file of SuperMC 
TABLE 3: Input data for the validation of dose optimization calculation.

\begin{tabular}{lc}
\hline Parameter name & Value \\
\hline Workers moving speed & $3600 \mathrm{~m} / \mathrm{h}$ \\
Operating time & $0.01 \mathrm{~h}$ \\
Room length & $20 \mathrm{~m}$ \\
Room width & $10 \mathrm{~m}$ \\
\hline
\end{tabular}

TABle 4: Dose results of four operation plans.

\begin{tabular}{lcccc}
\hline $\begin{array}{l}\text { Operating } \\
\text { point }\end{array}$ & $\begin{array}{c}\text { Route dose } \\
\text { (gone) }\end{array}$ & $\begin{array}{c}\text { Operation } \\
\text { dose }\end{array}$ & $\begin{array}{c}\text { Route dose } \\
\text { (leave) }\end{array}$ & $\begin{array}{c}\text { Total } \\
\text { dose }\end{array}$ \\
\hline B1 & 0.72 & 2.22 & 0.45 & 3.40 \\
B2 & 1.04 & 6.16 & 0.68 & 7.88 \\
B3 & 0.70 & 3.63 & 0.63 & 4.96 \\
B4 & 0.73 & 2.98 & 0.37 & 4.08 \\
\hline
\end{tabular}

Note: the unit is $\mathrm{mSv}$.

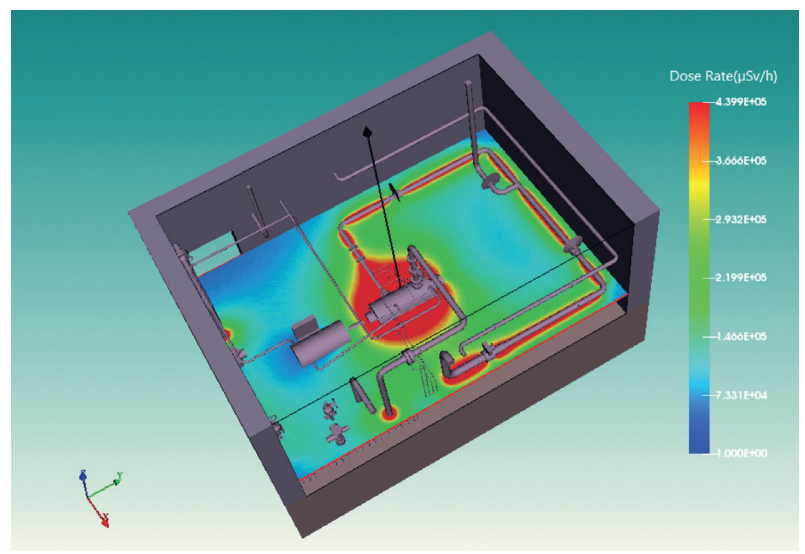

FIgURE 6: Example of fine 3D radiation field and display.

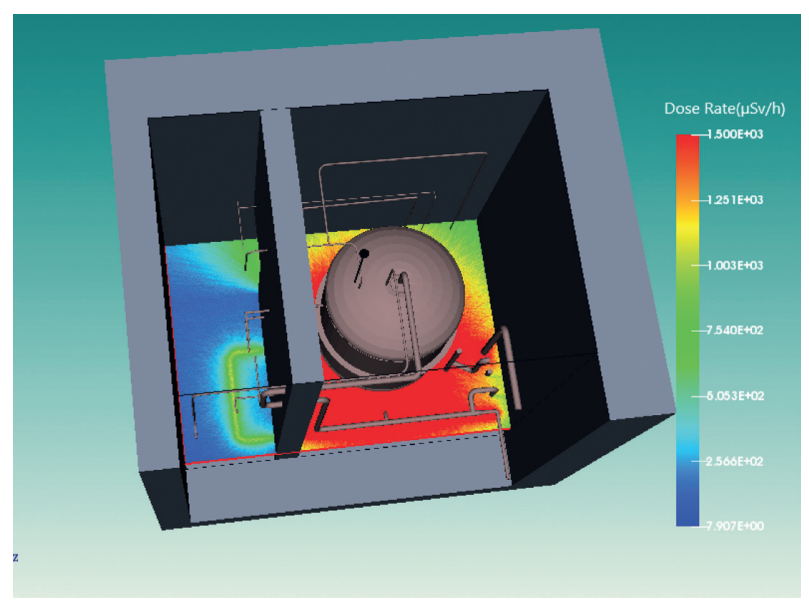

FIGURE 7: Shielding effect evaluation of labyrinth and door.

calculation based on the PDMS model. The interface modules in CPDS 2.0 and MCAM are used to prepare the source term input file. The total time needed for input file preparation and code computing is less than 3 hours with parallel computing.

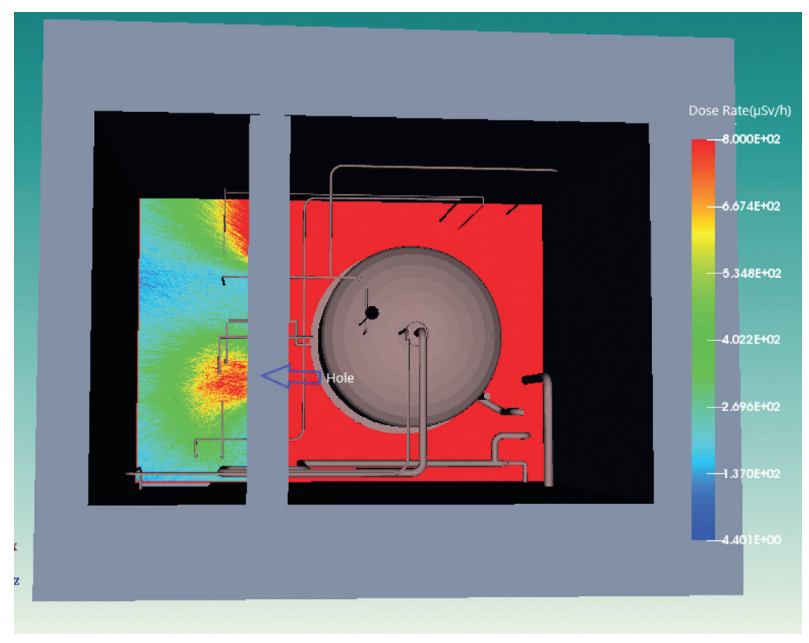

FIgURE 8: Shielding evaluation for the hole on the wall.

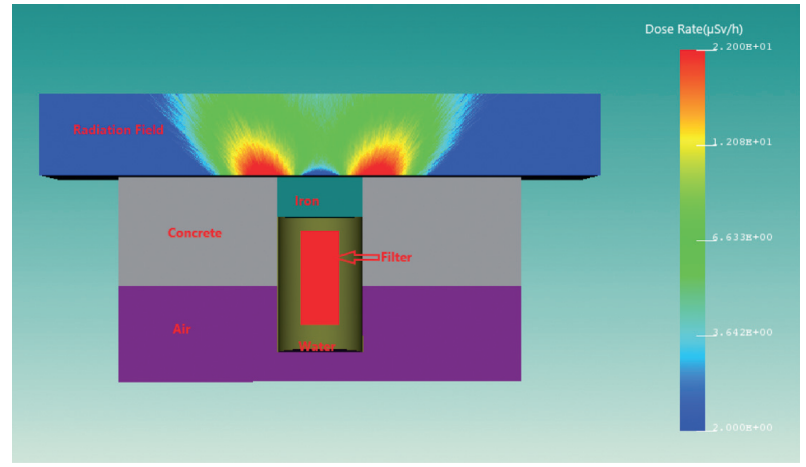

FIGURE 9: Shielding evaluation for the reactor coolant filter.

4.2. Shielding Evaluation of Labyrinth and Door. An example of the shielding evaluation of a labyrinth and a door in a typical radioactive compartment using the RPOS system is shown in Figure 7. The shielding effect of the labyrinth is clearly visible from the $3 \mathrm{D}$ radiation field, and the dose rate at the labyrinth opening is significantly reduced. It is also shown that the layout of pipes could be optimized as the current design leads to significant dose contribution in the labyrinth.

4.3. Inspection of Radiation Streaming and Shielding Weakness. 3D radiation field generated by the RPOS system can strongly facilitate the inspection of radiation streaming and shielding weakness. Figure 8 presents a radiation streaming due to the hole in the wall between the labyrinth and the equipment compartment. Figure 9 shows that the shielding scheme of the coolant filter cannot meet the design requirement, which means that the thickness of the concrete shielding wall on the side of the filter needs to be increased.

\section{Conclusions}

This paper describes the solutions of the three key technical issues of rapid 3D radiation field evaluation technique for the 
nuclear power plant, which inspire the development of the RPOS system. Tests of the RPOS system on existing in-service nuclear power plants in China demonstrate that the system can simulate the 3D radiation field rapidly and accurately and can be used directly on dose optimization of workers. The total time required for radiation field generation of a typical compartment in nuclear power plant can be limited to half day. Based on comparison of measurement data, the relative error of the reconstructed radiation field is below or around $30 \%$. The RPOS system has been applied in the HPR1000 on a large scale and significantly improves the efficiency of radiation protection design and enhances the ALARA assessment and justification. The RPOS system is also a powerful and efficient system with strong scalability. It can be widely used in radiation protection design of nuclear facilities, as well as decommissioning of nuclear facilities by extending the interfaces with various software, hardware, and database.

\section{Data Availability}

The data used to support the findings of this study are available from the corresponding author upon request.

\section{Conflicts of Interest}

The authors declare that there are no conflicts of interest regarding the publication of this paper.

\section{References}

[1] Y. Chen, Y. Wu, and Q. Huang, "Monte Carlo based operational and shutdown dose rate calculations of HT-7U tokamak," Fusion Engineering and Design, vol. 70, no. 2, pp. 155-162, 2004.

[2] Z. Tang, P. Long, S. Huang, J. Zou, and Y. Qiu, "Real-time dose assessment and visualization of radiation field for EAST tokamak," Fusion Engineering and Design, vol. 85, no. 7-9, pp. 1591-1594, 2010.

[3] F. Vermeersch, "ALARA pre-job studies using the VISIPLAN 3D ALARA planning tool," Radiation Protection Dosimetry, vol. 115, no. 1-4, pp. 294-297, 2005.

[4] X. George and S. Bushart, "Virtual-reality dose simulation for nuclear power plant," in Proceedings of the American Nuclear Society's 14th Biennial Topical Meeting of the Radiation Protection and Shielding Division, Carlsbad, NM, USA, April 2006.

[5] A. C. A. Mól, C. A. F. Jorge, P. M. Couto, S. C. Augusto, G. G. Cunha, and L. Landau, "Virtual environments simulation for dose assessment in nuclear plants," Progress in Nuclear Energy, vol. 51, no. 2, pp. 382-387, 2009.

[6] S. C. Augusto, A. C. A. Mol, C. A. F. Jorge et al., "Use of virtual reality to estimate radiation dose rates in nuclear plants," in Proceedings of the International Nuclear Atlantic ConferenceINAC 2007, Santos, Brazil, September 2007.

[7] J. Rodenas, "Developing a virtual reality application for training nuclear power plant operators: setting up a database containing dose rates in the refuelling plant," Radiation Protection Dosimetry, vol. 111, no. 2, pp. 173-180, 2004.

[8] Y. Ohga, M. Fukuda, K. Shibata, T. Kawakami, and T. Matsuzaki, "A system for the calculation and visualisation of radiation field for maintenance support in nuclear power plants," Radiation Protection Dosimetry, vol. 116, no. 1-4, pp. 592-596, 2005.

[9] Y. Iguchi, Y. Kanehira, M. Tachibana, and T. Johnsen, "Development of decommissioning engineering support system (DEXUS) of the fugen nuclear power station," Journal of Nuclear Science and Technology, vol. 41, no. 3, pp. 367-375, 2004.

[10] H. S. Park, S. K. Kim, K. W. Lee, C. H. Jung, and S. I. Jin, "Visualization of a dismantling environment for an evaluation of a worker's dose during the decommissioning of KRR-1\&2," Annals of Nuclear Energy, vol. 35, no. 6, pp. 1117-1124, 2008.

[11] K. Khattab, M. Boush, and H. Alkassiri, "Dose mapping simulation using the MCNP code for the Syrian gamma irradiation facility and benchmarking," Annals of Nuclear Energy, vol. 58, pp. 110-112, 2013.

[12] A. Serikov, U. Fischer, R. Heidinger, H. Tsige-Tamirat, and Y. Luo, "Radiation shielding analyses for the ITER upper port ECRH launcher," Fusion Science and Technology, vol. 53, no. 1, pp. 184-195, 2008.

[13] A. Serikov, U. Fischer, R. Heidinger, K. Kleefeldt, P. Spaeh, and D. Strauss, "Nuclear safety and waste management aspects of the EP ECRH upper launcher for ITER," Fusion Engineering and Design, vol. 84, no. 7-11, pp. 1751-1754, 2009.

[14] M. Zhang and S. Zou, "Inversion and construction of nuclear radiation field," Metallurgical and Mining Industry, vol. 8, pp. 78-85, 2015.

[15] W. Zhuang and J. Cai, "Inversion of radiation field on nuclear facilities: a method based on net function interpolation," Radiation Physics and Chemistry, vol. 153, pp. 27-34, 2018.

[16] Z. Wang and J. Cai, "Reconstruction of the neutron radiation field on nuclear facilities near the shield using Bayesian inference," Progress in Nuclear Energy, vol. 118, Article ID 103070, 2020.

[17] $\mathrm{Y} . \mathrm{Wu}$, "CAD-based interface programs for fusion neutron transport simulation," Fusion Engineering and Design, vol. 84, no. 7-11, pp. 1987-1992, 2009.

[18] Y. Wu, J. Song, H. Zheng et al., "CAD-based Monte Carlo program for integrated simulation of nuclear system SuperMC," Annals of Nuclear Energy, vol. 82, pp. 161-168, 2015.

[19] Ansys Incorporation, Free 3D Modeling Software:Ansys SpaceClaim Trial, Ansys Incorporation, Canonsburg, PA, USA, 2020, https://www.ansys.com/products/3d-design/ discovery-trial/spaceclaim.

[20] W. Lv, J. Xiong, and S. Tang, "Operation dose optimization study on three-dimensional dose field based on genetic algorithm," Chinese Journal of Nuclear Science and Engineering, vol. 37, pp. 206-212, 2017, in Chinese. 